\title{
AÇÃO TRANSOVARIANA DE LUFENURON (50 G/L) SOBRE ADULTOS DE Spodoptera frugiperda (J. E. Smith) (Lepidoptera: Noctuidae) E SEU EFEITO SOBRE O PARASITÓIDE DE OVOS Trichogramma pretiosum Riley (Hymenoptera: Trichogrammatidae)
}

\author{
Transovarian action of lufenuron on adults of Spodoptera frugiperda (J. E. Smith) \\ (Lepidoptera: Noctuidae) and its effect on the parasitoid Trichogramma pretiosum \\ Riley (Hymenoptera: Trichogrammatidae)
}

\author{
Dirceu Pratissoli ${ }^{1}$, Robson Thomaz Thuler $^{2}$, Fabrício Fagundes Pereira ${ }^{2}$, \\ Edvaldo Fialho dos Reis ${ }^{3}$, Andrea Torres Ferreira ${ }^{4}$
}

\section{RESUMO}

Avaliaram-se a ação transovariana do lufenuron em Spodoptera frugiperda e sua seletividade ao parasitóide de ovos Trichogramma pretiosum. Casais da praga foram isolados em gaiolas de PVC e alimentados com solução de mel a $10 \%$ na testemunha, e nos outros tratamentos, foi adicionado à solução de mel o regulador de crescimento de insetos Match ${ }^{\circledR}$ CE nas proporções de 12,$5 ; 15,0$ e 17,5 g i.a/l. Para verificação da ação transovariana, diariamente foram coletadas as posturas, contado o número de ovos e, posteriormente, o número de larvas eclodidas. Quarenta ovos provenientes de cada tratamento foram colados em cartelas de papel (cartolina) e expostos ao parasitismo, dentro de tubos de vidro de 1,0 x 3,5 cm, contendo uma fêmea de T. pretiosum no seu interior. Cartelas contendo 40 ovos de $S$. frugiperda foram imersas em soluções de lufenuron com a mesma concentração dos tratamentos anteriores e, posteriormente, expostas ao parasitismo por $T$. pretiosum. O lufenuron afetou consideravelmente a viabilidade dos ovos de $S$. frugiperda. Pelos resultados obtidos nos ensaios, relativos ao parasitóide, demonstram-se a seletividade do regulador de crescimento lufenuron e a possibilidade de sua utilização em programas de Manejo Integrado, juntamente com o parasitóide de ovos T. pretiosum.

Termos para indexação: Regulador de crescimento de insetos, seletividade, inseticida, lagarta-do-cartucho, milho.

\section{ABSTRACT}

The transovarian action of insect growth regulator lufenuron in Spodoptera frugiperda (Lepidoptera: Noctuidae) and its effects on the egg parasitoid Trichogramma pretiosum (Hymenoptera: Trichogrammatidae), were evaluated. Pest couples were isolated in PVC's cages and provided with 10\% honey solution as control. In the others treatments, the honey solution was mixed with the insect growth regulator Match ${ }^{\circledR} \mathrm{CE}$ using the following concentrations of $12.5 ; 15.0$ and 17.5 g i.a./l. For verification of the transovarian action, the eggs were collected daily, with the number of the hatched larvae being subsequently counted. Forty eggs from each treatment were fixed in paper cards and exposed to parasitism, inside clean vials of $1.0 \times 3.5 \mathrm{~cm}$, containing one female of the parasitoid. Cards with forty host eggs were dipped in lufenuron solutions with the same concentrations of the previous experiment, and these exposed to parasitism. Lufenuron affected considerably the viability of the host eggs. The results, regarding to the parasitoid, showed the selectivity of this insecticide and the possibility of its utilization in programs of Integrated Pest Management, with T. pretiosum.

Index terms: Insect growth regulator, selectivity, inseticide, fall armyworm, corn.

(Recebido para publicação em 25 de agosto de 2002 e aprovado em 13 de dezembro de 2002)

\section{INTRODUÇÃO}

A lagarta-do-cartucho do milho, Spodptera frugiperda, é uma praga de ocorrência em toda a
América e em algumas ilhas da Índia. No Brasil, ocorre durante todo o ano, em todas as regiões, devido à disponibilidade e diversidade de alimentação (CRUZ, 1995).

\footnotetext{
1. Engenheiro Agrônomo, Dr., Professor de Entomologia, Centro de Ciências Agrárias da UFES, Caixa Postal 16, 29500-000 - Alegre, ES. dirceu@npd.ufes.br

2. Engenheiro Agrônomo, Mestrando em Fitossanidade/Entomologia, UFRPE, Avenida Dom Manoel de Medeiros s/n, Dois Irmãos 52171-900 - Recife, PE. rthuler@zipmail.com

3. Engenheiro Agrícola, Dr., Professor de Irrigação e Drenagem, Centro de Ciências Agrárias da UFES, Caixa Postal 16, $29500-000$ - Alegre, ES. edfialho@npd.ufes.br

4. Engenheira Agrônoma, Mestranda em Entomologia, UENF - Lavras, MG.
} 
Sua relevância como praga vem aumentando gradativamente, principalmente devido ao desequilíbrio biológico, pela eliminação de seus inimigos naturais, além do aumento da exploração da cultura do milho, que é cultivada em várias regiões brasileiras, em duas safras anuais (CRUZ et al., 1999).

O controle químico é ainda o mais utilizado para a lagarta-do-cartucho, tendo aumentado atualmente o uso dos inseticidas pertencentes ao grupo dos reguladores de crescimento, como o Lufenuron (GRÜTZMACHER et al., 2000), que atua na síntese de quitina, alterando o processo de ecdise (GALLO et al., 2002). Esses inseticidas possuem alta especificidade, além de apresentarem baixa toxicidade para mamíferos (SCHROEDER et al., 1976; SCHROEDER e SUTTON, 1978; GROSSCURT, 1978). Atuam também sobre os adultos, que se contaminam ao se alimentar, provocando esterilidade dos mesmos e reduzindo sua fecundidade, além de afetar a viabilidade dos ovos (ÁVILA e NAKANO, 1999).

A ocorrência natural de predadores e parasitóides, nos agroecossistemas, é um fator de grande importância para a redução da infestação da praga em plantios de milho. Destacam-se entre esses inimigos naturais o predador Doru luteipes e os parasitóides Telenomus sp., Chelonus insularis, Campoletis flavicincta e Trichogramma sp. (CRUZ, 1995), sendo espécies desse último gênero utilizadas atualmente em 32 milhões de hectares em mais de 30 países (LI, 1994).

A presença desses inimigos naturais em campos produtores de milho torna essencialmente criteriosa a escolha de produtos químicos para utilização no controle de pragas, viabilizando, assim, o uso de produtos como os reguladores de crescimento devido à sua especificidade e seletividade aos inimigos naturais (GRÜTZMACHER et al., 2000).

A seletividade, segundo Cruz (1995), é a chave do Manejo de Pragas em sistemas que visam a reduzir a população de insetos nocivos, sem alterar ou promovendo o mínimo possível de alteração em outros componentes do agroecossistema e do ambiente de uma maneira geral. Assim, com este trabalho objetivou-se avaliar a existência de ação transovariana do Lufenuron $\left(\right.$ Match $^{\circledR} \mathrm{CE}$ ) em adultos de $S$. frugiperda e sua seletividade ao parasitóide de ovos Trichogramma pretiosum.

\section{MATERIAL E MÉTODOS}

O trabalho foi realizado no Laboratório de Entomologia do Centro de Ciências Agrárias da Universidade Federal do Espírito Santo (CCA-UFES), localiza- do na cidade de Alegre, em sala climatizada com temperatura de $25 \pm 1^{\circ} \mathrm{C}$, umidade relativa de $70 \pm 5 \%$ e fotofase de 14 horas.

\section{Criação Massal}

Os insetos utilizados na experimentação foram obtidos da criação massal de $S$. frugiperda, cuja fase jovem foi mantida em dieta artificial estabelecida por Kasten Júnior et al. (1978), individualizando-se as larvas em tubos de vidro de $2,5 \times 8,5 \mathrm{~cm}$. A fase adulta do inseto foi mantida em gaiolas de PVC com 24,5 x 19,5 $\mathrm{cm}$, revestidas internamente por papel-ofício para facilitar coleta dos ovos. As gaiolas são fechadas na extremidade superior com tela do tipo 'filó' e no seu interior é colocada uma placa de Petri com as pupas dos insetos, próximos à emergência. Sobre a placa de Petri, foi colocado um frasco plástico contendo solução de mel a $10 \%$, fechado com um chumaço de algodão em forma de pavio, para alimentação dos insetos.

\section{Ação transovariana}

Para avaliação do efeito do Lufenuron sobre os adultos, foram sexados, ainda na fase de pupa, 48 casais de $S$. frugiperda, os quais foram isolados em gaiolas idênticas às descritas na metodologia de criação desse inseto; no entanto, com tamanho de $10 \times 10 \mathrm{~cm}$.

$\mathrm{O}$ delineamento experimental utilizado foi $\mathrm{o}$ inteiramente casualisado com 4 tratamentos e 12 repetições, sendo os insetos alimentados com solução de mel a $10 \%$ na testemunha e adicionando-se 12,$5 ; 15,0$ e $17, \mathrm{~g}$ i.a/l de lufenuron $\left(\right.$ Match $^{\circledR} \mathrm{CE}$ ) à solução de mel para o primeiro, segundo e terceiro tratamentos, respectivamente. $\mathrm{O}$ alimento foi renovado a cada 3 dias, mantendo-se os mesmos tratamentos.

A partir do terceiro dia de acasalamento, foi realizada a coleta dos ovos, diariamente, até o término do período de oviposição. Os ovos coletados foram acondicionados em "gerbox" e transferidos para câmaras climatizadas (BOD) com temperatura, umidade relativa e fotofase idênticas às da sala climatizada.

Com a eclosão as larvas, essas foram contadas e retiradas do recipiente, para evitar a destruição dos ovos restantes. Esse processo foi repetido durante 5 dias e foram avaliados o número de ovos viáveis, a longevidade dos adultos e o número total de ovos.

\section{Efeito do resíduo nos Ovos sobre Trichogramma}

Ovos provenientes dos casais de $S$. frugiperda individualizados e tratados com as diferentes dosagens

Ciênc. agrotec., Lavras, v. 28, n. 1, p. 9-14, jan./fev., 2004 
de Lufenuron no primeiro experimento foram coletados e colados, quarenta por cartela de $0,8 \times 3,0$ $\mathrm{cm}$. Essas cartelas foram inseridas em tubos de vidro de 1,0 x 3,5 cm, contendo uma fêmea do parasitóide, permitindo o parasitismo por um período de 24 horas.

O delineamento experimental utilizado foi o inteiramente casualisado com 4 tratamentos e 20 repetições, sendo avaliadas a porcentagem de parasitismo, a viabilidade e a razão sexual de $T$. pretiosum.

\section{Seletividade do Lufenuron ao Parasitóide}

Neste experimento, cartelas idênticas às descritas no experimento anterior foram imersas em soluções de lufenuron nas concentrações de 12,5; 15,0 e 17,g i.a/l e em água destilada (testemunha). Posteriormente, as cartelas foram introduzidas em tubos de vidro de 1,0 x 3,5 $\mathrm{cm}$ contendo uma fêmea de $T$. pretiosum. Após 24 horas de parasitismo, as fêmeas foram descartadas, e as cartelas, mantidas nos tubos até a emergência do parasitóide.

Este experimentou constou de 4 tratamentos e 15 repetições e foram avaliados o parasitismo, a razão sexual e a viabilidade de $T$. pretiosum.

Os dados obtidos na experimentação foram submetidos à análise de variância, e as médias, comparadas pelo teste de Tukey $(\mathrm{P} \leq 0,05)$.

\section{RESULTADOS E DISCUSSÃO}

\section{Ação transovariana}

O regulador de crescimento Lufenuron (Match ${ }^{\circledR} \mathrm{CE}$ ), quando ingerido pelas fêmeas de $S$. frugiperda, não afetou a fecundidade das mesmas, representada pelo número total médio de ovos, nem a longevidade média (Tabela 1); no entanto, a viabilidade média dos ovos foi alterada consideravelmente, por todas as dosagens empregadas no experimento, podendo-se verificar que a dosagem recomendada pelo fabricante - tratamento 2 - T2 (4,75\%) foi a que mais reduziu a viabilidade média dos ovos: uma redução de $93 \%$ em relação à viabilidade média da testemunha, seguida pela superdosagem - tratamento 3 - T3, que apresentou $13,7 \%$ e pela subdosagem - tratamento 1 - T1, com 18,13\% de viabilidade média (Tabela 1).

A ação transovariana do lufenuron pode ser constatada tanto por esses resultados quanto pelo desenvolvimento embrionário, observado através do córion dos ovos provenientes dos tratamentos, uma vez que, após 3 - 4 dias, os mesmos apresentavam-se "murchos", e em poucos deles notava-se a formação do embrião.

Ávila e Nakano (1999) observaram ação transovariana do regulador de crescimento lufenuron em Diabrotica speciosa, caracterizada pela reduzida eclosão das larvas, apesar de ter sido observado pelos autores o desenvolvimento embrionário, resultado esse semelhante ao constatado por Schroeder e Sutton (1978), que observaram menor viabilidade de ovos de Diaprepes abbreviatus, quando os adultos foram alimentados com folhas de citrus impregnadas com o regulador de crescimento diflubenzuron.

\section{Efeito do Resíduo nos Ovos, para o Parasitóide}

Os ovos provenientes dos tratamentos e expostos ao $T$. pretiosum apresentaram reduzido parasitismo, diferindo estatisticamente da testemunha em todos os tratamentos (Tabela 2). A viabilidade dos descendentes foi afetada apenas pela superdosagem (T3), que apresentou $80,7 \%$ de emergência; no entanto, não se pode considerar tão relevante tal redução, se comparada à taxa de emergência de $89 \%$, apresentada pela testemunha (Tab. 2). A razão sexual dos descendentes não foi alterada, indicando esses parâmetros baixa ação dos resíduos do inseticida sobre o terceiro nível trófico; nesse caso, o parasitóide de ovos $T$. pretiosum. Takada et al. (2001) verificaram resultado semelhante em relação à viabilidade da progênie de Trechogramma dendrolimi, em ovos de Mamestra brassicae, tratados com o regulador de crescimento clorfluazuron. 
TABELA 1 - Número total de ovos, viabilidade e longevidade de adultos de Spodoptera frugiperda, oriundos dos tratamentos com lufenuron e testemunha.

\begin{tabular}{ccccc}
\hline \multirow{2}{*}{ Tratamento } & $\begin{array}{c}\text { Número total médio } \\
\text { de ovos }\end{array}$ & Viabilidade $(\%)$ & Machos & Fêmeas \\
\cline { 3 - 5 } & $1484,84 \mathrm{a}$ & $68,84 \mathrm{a}$ & $14,00 \mathrm{a}$ & $11,30 \mathrm{a}$ \\
Testemunha & $1325,53 \mathrm{a}$ & $18,13 \mathrm{~b}$ & $13,92 \mathrm{a}$ & $11,76 \mathrm{a}$ \\
I & $1377,61 \mathrm{a}$ & $04,75 \mathrm{c}$ & $15,23 \mathrm{a}$ & $15,76 \mathrm{a}$ \\
II & $1649,15 \mathrm{a}$ & $13,07 \mathrm{~b}$ & $13,92 \mathrm{a}$ & $15,15 \mathrm{a}$ \\
\hline
\end{tabular}

Médias seguidas de mesma letra na coluna não diferem entre si pelo teste de Tukey a $5 \%$ de significância.

TABELA 2 - Parasitismo, viabilidade e razão sexual de Trichogramma pretiosum em ovos oriundos de casais de Spodoptera frugiperda, alimentados com mel e diferentes dosagens de lufenuron $(50 \mathrm{~g} / \mathrm{l})$.

\begin{tabular}{cccc}
\hline Tratamento & $\begin{array}{c}\text { Parasitismo } \\
(\boldsymbol{\%})\end{array}$ & $\begin{array}{c}\text { Emergência } \\
(\%)\end{array}$ & $\begin{array}{c}\text { Razão } \\
\text { sexual }\end{array}$ \\
\hline Testemunha & $59,5 \mathrm{a}$ & $89,0 \mathrm{a}$ & $0,71 \mathrm{a}$ \\
I & $38,8 \mathrm{~b}$ & $95,7 \mathrm{a}$ & $0,69 \mathrm{a}$ \\
II & $43,2 \mathrm{~b}$ & $96,3 \mathrm{a}$ & $0,61 \mathrm{a}$ \\
III & $43,0 \mathrm{~b}$ & $80,7 \mathrm{~b}$ & $0,63 \mathrm{a}$ \\
\hline
\end{tabular}

Médias seguidas de mesma letra na coluna não diferem entre si pelo teste de Tukey a $5 \%$ de significância.

\section{Seletividade ao Parasitóide}

Quando os ovos de S. frugiperda foram tratados por imersão nas soluções de Lufenuron, em superdosagem (T3), observaram-se redução do parasitismo e aumento da razão sexual do parasitóide, tendo esse tratamento apresentado diferença estatística significativa em relação aos demais. A emergência dos adultos só não foi alterada quando aplicou-se a subdosagem (T1), que não diferiu da testemunha. No entanto, na dosagem comercial (T2), o inseticida não ocasionou grande alteração nesse parâmetro (Tabela 3).

Carvalho et al. (1994), em teste com diversos reguladores de crescimento, também não observaram alteração no número de ovos parasitados e na razão sexual de T. pretiosum.
À semelhança desses resultados, Suh et al. (2000) não observaram alteração na porcentagem de emergência e razão sexual de Trechogramma exiguum, em relação à testemunha, quando ovos de Helicoverpa zea, parasitados, foram imersos nas caldas de methoxyfenozide e tebufenozide em suas concentrações comerciais.

TABELA 3 - Parasitismo, viabilidade e razão sexual de Trichogramma pretiosum em ovos de Spodoptera frugiperda, tratados com diferentes dosagens de lufenuron $(50 \mathrm{~g} / 1)$ e testemunha.

\begin{tabular}{cccc}
\hline Tratamento & $\begin{array}{c}\text { Parasitismo } \\
\text { (\%) }\end{array}$ & $\begin{array}{c}\text { Emergên- } \\
\text { cia (\%) }\end{array}$ & $\begin{array}{c}\text { Razão } \\
\text { sexual }\end{array}$ \\
\hline Testemunha & $57,8 \mathrm{a}$ & $79,0 \mathrm{a}$ & $0,68 \mathrm{~b}$ \\
I & $68,0 \mathrm{a}$ & $59,7 \mathrm{a}$ & $0,61 \mathrm{~b}$ \\
II & $61,0 \mathrm{a}$ & $51,4 \mathrm{~b}$ & $0,69 \mathrm{~b}$ \\
III & $40,5 \mathrm{~b}$ & $39,2 \mathrm{c}$ & $0,92 \mathrm{a}$ \\
\hline
\end{tabular}

Médias seguidas de mesma letra na coluna não diferem entre si pelo teste de Tukey a 5\% de significância.

Os resultados obtidos neste trabalho em relação à seletividade do lufenuron ao parasitóide de ovos $T$. pretiosum são coincidentes com os observados por Ciociola Júnior et al. (1999), para os reguladores de crescimento diflubenzuron, teflubenzuron, triflumuron e tebufenozide; por Carvalho et al. (2001), para clorfluazuron, teflubenzuron, triflumuron e tebufenozide e por Cônsoli et al. (1998), para tebufenozide em todos os estágios imaturos de $T$. pretiosum e para teflubenzuron, nos estágios de pré-pupa e pupa, do mesmo parasitóide. 


\section{CONCLUSÕES}

a) Ficou comprovada a ação transovariana do Lufenuron (Match ${ }^{\circledR} \mathrm{CE}$ ), constatada pela considerável redução na viabilidade dos ovos de $S$. frugiperda provenientes de adultos tratados com o princípio ativo.

b) O princípio ativo Lufenuron mostrou-se uma boa opção para utilização em programas de Manejo Integrado de Pragas, tendo em vista a seletividade apresentada ao parasitóide $T$. pretiosum, em todos os testes, principalmente na dosagem recomendada do produto.

\section{REFERÊNCIAS BIBLIOGRÁFICAS}

ÁVILA, C. J.; NAKANO, O. Efeito do regulador de crescimento lufenuron na reprodução de Diabrotica speciosa (Germar) (Coleoptera: Chrysomelidae). Anais da Sociedade Entomológica do Brasil, Londrina, v. 28, n. 2, p. 293-299, jun. 1999.

CARVALHO, G. A.; PARRA, J. R. P.; BAPTISTA, G. C. Seletividade de alguns produtos fitossanitários a duas linhagens de Trichogramma pretiosum Riley, 1879 (Hymenoptera: Trichogrammatidae). Ciência e Agrotecnologia, Lavras, v. 25, n. 3, p. 583-591, maio/jun. 2001 .

CARVAlHO, G. A.; TIRONI, P.; RIGITANO, R. L. O.; SALGADO, L. O. Seletividade de inseticidas reguladores de crescimento de insetos à Trichogramma pretiosum Riley (Hymenoptera: Trichogrammatidae). Anais da Sociedade Entomológica do Brasil, Londrina, v. 23, n. 3, p. 431-434, 1994.

CIOCIOLA JÚNIOR, A. I.; DINIZ, L. D.; ZACARIAS, M. S.; CARVALHO, A. R.; CIOCIOLA, A. I. Impacto de inseticidas sobre a emergência de Trichogramma pretiosum, Riley (Hymenoptera: Trichogrammatidae). Ciência e Agrotecnologia, Lavras, v. 23, n. 3, p. 589592, jul./set. 1999.

CÔNSOLI, F. L.; PARRA, J. R. P.; HASSAN, S. A. Side-effects of insecticides used in tomato fields on the egg parasitoid Trichogramma pretiosum Riley (Hym., Trichogrammatidae) a natural enemy of Tuta absoluta (Meyrick) (Lep., Gelechiidae). Journal of Applied Entomology, College Park, v. 122, p. 43-47, 1998.

CRUZ, I. A lagarta-do-cartucho na cultura do milho. Sete Lagoas: EMBRAPA-CNPMS, 1995. 45 p. (Circular Técnica, 21).
CRUZ, I.; FIGUEIREDO, M. L. C.; MATOSO, M. J. Controle biológico de Spodoptera frugiperda utilizando o parasitóide de ovos Trichogramma. Sete Lagoas: EMBRAPA-CNPMS, 1999. 40 p. (Circular Técnica, 30).

GALLO, D.; NAKANO, O.; SILVEIRA NETO, S.; CARVALHO, R. P. L.; BAPTISTA, G. C.; BERTI FILHO, E.; PARRA, J. R. P.; ZUCHI, R. A.; ALVES, S. B.; VENDRAMIM, J. D.; MARCHINI, L. C.; LOPES, J. R. S.; OMOTO, C. Entomologia Agrícola. 3. ed. Piracicaba: FEALQ, 2002. 920 p.

GROSSCURT, A. C. Diflubenzuron: some aspects of ists ovicidal and larvicidal mode of action and evaluation of its practical possibilities. Pesticide Science, London, v. 9, p. 373-386, 1978.

GRÜTZMACHER, A. D.; MARTINS, J. F. S.; AZEVEDO, R.; GIOLO, F. P. Efeito de inseticidas e de tecnologias de aplicação no controle da lagarta-docartucho na cultura do milho no agroecossistema de várzea. In: REUNIÃO TÉCNICA ANUAL DO MILHO, 45.; REUNIÃO TÉCNICA ANUAL DO SORGO, 28., 2000, Pelotas. Anais... Pelotas: EMBRAPA-CPACT, 2000. p. 567-573.

KASTEN JÚNIOR, P.; PRECETI, A. C. M.; PARRA, J. R. P. Dados biológicos comparativos de Spodoptera frugiperda (J. E. Smith, 1797) em duas dietas artificiais e substrato natural. Revista de Agronomia, Piracicaba, v. 53, n. 1/2, p. 68-78, 1978.

LI, L. Y. Worldwide use of Trichogramma for biological control on different crops: a survey. In: WAJNBERG, E.; HASSAN, S. A. (Eds.). Biological control with egg parasitoids. London: Biddles, 1994. p. 37-53.

SCHROEDER, W. J.; BEAVERS, J. B.; SUTTON, R. A.; SELHIME, A. G. Ovicidal effect of Thompson-Hayward TH 6040 in Diaprepes abbreviatus on citrus in Florida. Journal of Economic Entomology, Maryland, v. 69, n. 6, p. 780-782, Dec. 1976.

SCHROEDER, W. J.; SUTTON, R. A. Diaprepes abbreviatus: suppression of reproductive potential on citrus with an insect regulator plus spray oil. Journal of Economic Entomolology, Maryland, v. 71, n. 1, p. 69-70, Jan. 1978. 
SUH, C. P. C.; ORR, D. B.; DUYN, J. W. van. Effect of insecticides on Trichogramma exiguum (Hymenoptera: Trichogrammatidae) preimaginal development and adult survival. Journal of Economic Entomology, Maryland, v. 93, n. 3, p. 577-583, June 2000.
TAKADA, Y.; KAWAMURA, S.; TANAKA, T. Effects of various insecticides on the development of the egg parasitoid Trichogramma dendrolimi (Hymenoptera: Trichogrammatidae). Journal of Economic Entomology, Maryland, v. 94, n. 6, p. 1341-1343, Dec. 2001. 